\title{
What makes one person paranoid and another person anxious? The differential prediction of social anxiety and persecutory ideation in an experimental situation
}

\author{
D. Freeman ${ }^{1 *}$, M. Gittins ${ }^{2}$, K. Pugh ${ }^{1}$, A. Antley ${ }^{3}$, M. Slater ${ }^{3}$ and G. Dunn ${ }^{2}$ \\ ${ }^{1}$ Department of Psychology, Institute of Psychiatry, King's College London, UK \\ ${ }^{2}$ Biostatistics Group, School of Medicine, University of Manchester, UK \\ ${ }^{3}$ Department of Computer Science, University College London, UK
}

\begin{abstract}
Background. In recent years a close association between anxiety and persecutory ideation has been established, contrary to the traditional division of neurosis and psychosis. Nonetheless, the two experiences are distinct. The aim of this study was to identify factors that distinguish the occurrence of social anxiety and paranoid thoughts in an experimental situation.
\end{abstract}

Method. Two hundred non-clinical individuals broadly representative of the UK general population were assessed on a range of psychological factors, experienced a neutral virtual reality social environment, and then completed state measures of paranoia and social anxiety. Clustered bivariate logistic regressions were carried out, testing interactions between potential predictors and the type of reaction in virtual reality.

Results. The strongest finding was that the presence of perceptual anomalies increased the risk of paranoid reactions but decreased the risk of social anxiety. Anxiety, depression, worry and interpersonal sensitivity all had similar associations with paranoia and social anxiety.

Conclusions. The study shows that social anxiety and persecutory ideation share many of the same predictive factors. Non-clinical paranoia may be a type of anxious fear. However, perceptual anomalies are a distinct predictor of paranoia. In the context of an individual feeling anxious, the occurrence of odd internal feelings in social situations may lead to delusional ideas through a sense of 'things not seeming right'. The study illustrates the approach of focusing on experiences such as paranoid thinking rather than diagnoses such as schizophrenia.

Received 10 January 2008; Revised 10 April 2008; Accepted 15 April 2008; First published online 4 June 2008

Key words: Anxiety, delusions, paranoia, schizophrenia, virtual reality.

\section{Introduction}

In the past 10 years the importance of emotion in understanding psychosis has been increasingly recognized (Birchwood, 2003; Freeman \& Freeman, 2008). The divide between psychosis and neurosis has been narrowed. In particular, a close association between anxiety and paranoia has been demonstrated. Anxiety has repeatedly been found to be associated with paranoid thoughts (e.g. Martin \& Penn, 2001; Johns et al. 2004) and persecutory delusions (e.g. Freeman \& Garety, 1999; Startup et al. 2007). Anxiety is predictive of the occurrence of paranoid thoughts (Freeman et al. 2003, 2005a; Valmaggia et al. 2007) and the persistence of persecutory delusions

* Address for correspondence: Dr D. Freeman, Department of Psychology, PO Box 77, Institute of Psychiatry, King's College

London, Denmark Hill, London SE5 8AF, UK.

(Email: D.Freeman@iop.kcl.ac.uk)
(Startup et al. 2007). There is also emerging evidence that paranoid thoughts build upon common social anxieties such as fear of rejection (Freeman et al. $2005 a, b)$. A key impetus of this research has been to normalize psychotic experience and make it understandable. Nonetheless, paranoia and social anxiety are distinct experiences and differences in their causes need to be identified.

\section{Studying persecutory ideation}

There is a growing consensus that psychotic symptoms such as delusions are on a continuum with normal experience (e.g. Strauss, 1969; Chapman \& Chapman, 1980; Claridge, 1997; Van Os \& Verdoux, 2003). This view is based upon three lines of empirical evidence: the results of epidemiological surveys demonstrating that delusional ideation is not confined to psychotic groups (e.g. Eaton et al. 1991; Van Os et al. 2000); evidence of 'aetiological continuity' 
between non-clinical and clinical experiences (MyinGermeys et al. 2003); and findings that the risk of clinical disorder is increased by the earlier presence of low-level symptoms (Chapman et al. 1994; Poulton et al. 2000). Although complete discontinuity between clinical and non-clinical experiences is unlikely, the exact nature of a paranoia spectrum remains to be established. If delusions are caused by a number of interacting factors then it is unlikely that there will be a normal distribution in the general population (see Van Os \& Verdoux, 2003). Instead, the distribution in the general population is likely to be skewed, with many people not having any delusional experiences (i.e. quasi-continuous). At an individual level there may also be non-linear shifts into clinical disorder. Nonetheless, the important implication for researchers is that studying non-clinical delusional ideation can inform the understanding of clinical phenomena, just as studying anxious or depressive states can inform the understanding of emotional disorders.

The study of persecutory ideation is beset by the problem of justified suspicions (Freeman, 2008b). This is a particular problem in investigating nonclinical phenomena. Individuals can experience real hostility from others, and paranoia questionnaires cannot rule out thoughts that are grounded in reality. Even using an interview assessment, it can be very difficult to establish the truth of the claims underlying a suspicious thought. Therefore, an experimental method has been pioneered to study paranoid thinking (Freeman et al. 2003; Freeman, 2008a). This makes use of human responses being consistent between real and virtual worlds (Sanchez-Vives \& Slater, 2005). Virtual reality is used to present individuals with neutral social environments (e.g. a library, train carriage). It has been found that individuals' interpretations of the same environment vary greatly, from the positive to the negative, providing a striking illustration of the importance of appraisals in the experience of events. The key advantage of this method is that any paranoid thoughts that occur are known to be unfounded because the computer characters are not programmed to be hostile and behave in ways deemed by consensus to be neutral. No matter what a person does, the characters remain neutral in their responses.

\section{The psychological understanding of paranoia}

In the first large-scale study of the virtual reality method we tested 200 non-clinical individuals broadly representative of the UK population (Freeman et al. 2008). Predictors of paranoia were examined based upon a cognitive model of persecutory delusions
(Freeman et al. 2002; Freeman, 2007; Garety et al. 2007; Freeman \& Freeman, 2008). In the model it is hypothesized that individuals prone to paranoid ideation are trying to make sense of feelings of oddness caused by internal anomalies (e.g. hallucinations, perceptual anomalies, arousal). The causes of anomalies of experience include core cognitive dysfunction (e.g. Hemsley, 2005), impairment in earlystage sensory processing (e.g. Butler \& Javitt, 2005), illicit drug use (e.g. D'Souza et al. 2004), hearing impairment (e.g. Zimbardo et al. 1981) and dopamine dysregulation (e.g. Kapur, 2003). A persecutory interpretation of the anomalies is likely to be formed in the context of negative affect. Suspicious thoughts are often preceded by stressful events (e.g. difficult interpersonal relationships, bullying, isolation). The stresses tend to happen against a background of anxiety, worry and related interpersonal concerns. It is hypothesized that anxiety is central in the threat (mis)interpretation of the internal events. The final piece of the puzzle is reasoning. Ideas of a persecutory content are more likely to become of a delusional intensity when there are accompanying biases in reasoning such as reduced data gathering ('jumping to conclusions') (Garety \& Freeman, 1999; Van Dael et al. 2006) and a failure to consider alternative explanations (Freeman et al. 2004). Thus, emphasized in the psychological understanding of persecutory ideation are: anomalous experiences, which may be caused by core cognitive dysfunction and street drug use; affective processes, especially anxiety, worry and interpersonal sensitivity; reasoning biases, particularly belief confirmation, jumping to conclusions and belief inflexibility; and social factors, such as isolation and trauma. In the virtual reality study, key factors in the model - anxiety, worry, perceptual anomalies and cognitive flexibility - were all shown to predict paranoid reactions.

\section{The differential prediction of social anxiety and paranoia}

Social anxiety caused by the neutral social situation was also assessed in the general population study. Participants completed the Social Avoidance and Distress Scale (SAD) in relation to their experience of the virtual environment (Watson \& Friend, 1969). In this paper we report the factors that differentially predict the occurrence of social anxiety and paranoid thoughts in virtual reality. Although similarities between the two experiences are becoming evident, differences are of equal theoretical and clinical interest. Based on the persecutory delusions model, there was an a priori prediction that anomalies of experience would distinguish the prediction of paranoia and 


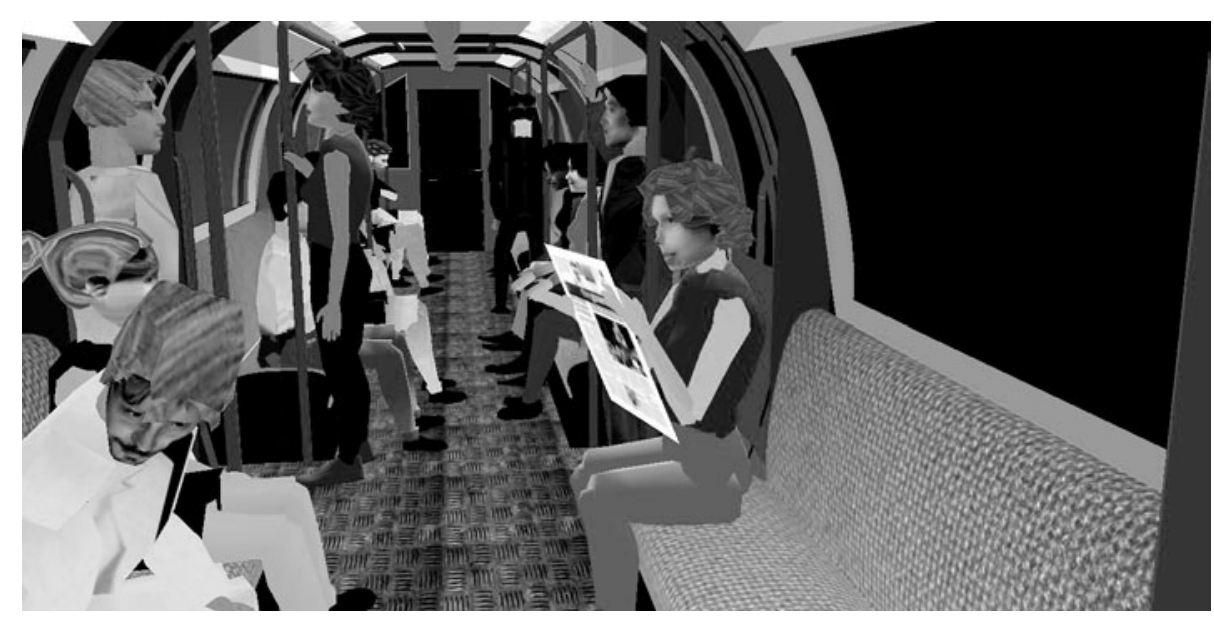

Fig. 1. The virtual reality underground train.

social anxiety. Entering a social situation when anxious will produce anxiety, but entering a social situation when anxious and having anomalies of experience will increase the likelihood of paranoid thinking. By contrast, it was expected that anxiety, depression, worry, interpersonal sensitivity and negative beliefs about the self would not distinguish the prediction of social anxiety and paranoia (i.e. affective processes contribute to the occurrence of both experiences). It was also hypothesized that reasoning style might be a differential predictor, but that this may be difficult to detect in a non-clinical study where the ability to correct interpretations may be protective against the development of clinical experiences.

\section{Method}

The procedure for each participant involved completion of a comprehensive psychological assessment, 5 minutes in an underground train virtual environment, followed by assessment of the experience.

\section{Participants}

A sample of the adult local population was recruited. A leaflet advertising a study of 'people's reactions in virtual reality' at the university was sent to all households in local postcodes. Participants were not informed before testing that the study was of paranoia or social anxiety. The main inclusion criterion was that participants were aged $\geqslant 18$ years. Potential participants were asked whether they had ever had a mental illness, been admitted to a psychiatric hospital, or been prescribed medication for such problems. Individuals reporting a history of severe mental illness (e.g. schizophrenia, bipolar disorder, affective psychosis) were excluded from the study. Individuals with a history of epilepsy were also excluded because of potential side-effects of virtual reality. Seven individuals with a history of severe mental illness and two individuals with a history of epilepsy were excluded. One hundred male and 100 female participants were recruited. They were paid $£ 20$ for their time. The occupationally based National Statistics Socio-economic Classification was used to categorize participants (Office for National Statistics, 2005). The study had received approval from the local research ethics committee.

\section{Virtual reality}

The head-mounted display used was a Virtual Research VR1280, which has a resolution of $1280 \times$ 1024 in each eye, a $60^{\circ}$ diagonal field of view and a refresh rate of $60 \mathrm{~Hz}$. The tracking system used for the scenario was the Intersense IS900. The tracker uses a hybrid of inertial and ultrasonic sensors to determine the orientation and position of the user during the simulation. The sensors were laid out in a ceiling constellation grid above the user. The tracker data were accessed by a Virtual Reality Peripheral Network (VRPN) IS900 server.

The virtual reality environment comprised a 5-minute journey between two stops on an underground train that was populated by computer characters (see Fig. 1). The Distributed Immersive Virtual Environment (DIVE) software platform was used to create the overall scenario (Frecon et al. 2001). Both the train shell and the computer characters ('avatars') were created using 3D Studio Max. The avatar motions were made using an optical motion capture system. Each avatar had its own background motion that repeated throughout the scenario. Each avatar had one motion that approximated their breath and another motion that randomized the 
direction of their gaze. In addition, several of the avatars responded to participants' gaze by looking in their direction. One avatar would occasionally smile at the user when looked at. The audio for the scene, comprising background tube noise and low-level snippets of conversation, was rendered in stereo, without spatialization, using a Creative sound card.

\section{Measures}

Before entering the virtual environment participants completed a battery of assessments.

\section{Intellectual functioning}

Wechsler Abbreviated Scale of Intelligence (WASI; Wechsler, 1999). The WASI is a nationally standardized short and reliable measure of intelligence linked to the Wechsler Adult Intelligence Scale-Third Edition (WAIS-III; Wechsler, 1997). The Vocabulary and Matrix Reasoning subtests were used in the current study.

\section{Emotional processes}

Depression Anxiety Stress Scales (DASS; Lovibond $\mathcal{E}$ Lovibond, 1995). The DASS is a 42-item instrument with three subscales measuring current symptoms of depression, anxiety and stress. Each of the subscales consists of 14 items with a $0-3$ scale $(0=$ did not apply to me at all, $3=$ applied to me very much). Higher scores indicate higher levels of emotional distress. The scale has been shown to be reliable and valid in a large UK non-clinical population (Crawford \& Henry, 2003). The anxiety and depression subscales were used in the current study.

Penn State Worry Questionnaire (PSWQ; Meyer et al. 1990). The PSWQ is the most established measure of trait worry style and has been used in non-clinical and clinical populations (see review by Startup \& Erickson, 2006). It assesses the tendency to worry but not the content of the thoughts. Each of the 16 items is rated on a five-point scale. Higher scores indicate a greater tendency to worry.

Worry Domains Questionnaire (WDQ; Tallis et al. 1992). The WDQ assesses the occurrence of a range of common (non-paranoid) worries (i.e. in contrast to the PSWQ, the scale assesses content). It has good psychometric properties (see Startup \& Erickson, 2006). The scale contains 25 items using a five-point rating scale (from not at all to extremely). Higher scores indicate greater levels of worry.
Catastrophizing interview (Vasey $\mathcal{E}$ Borkovec, 1992). The catastrophizing interview is an experimental assessment of worry style (see review of procedures by Davey, 2006). Individuals are asked what worries them about their main worry and this question is repeated for all their subsequent answers. The procedure is terminated when no further responses are given (i.e. the person can think of no more worries in the chain). Each answer is counted as a catastrophizing step. Increasing numbers of catastrophizing steps indicate a greater worry style.

Brief Core Schema Scales (Fowler et al. 2006). This measure, developed with non-clinical and psychosis groups, has 24 items each rated on a five-point scale (0-4). Four subscale scores are derived: negative beliefs about self, positive beliefs about self, negative beliefs about others and positive beliefs about others. Higher scores reflect greater endorsement of items.

Interpersonal Sensitivity Measure (Boyce E Parker, 1989). This 36-item scale was designed to assess interpersonal sensitivity defined as undue and excessive awareness of, and sensitivity to, the behaviour and feelings of others. Self-statements are rated on a fourpoint scale ( $1=$ very unlike self, $2=$ moderately unlike self, $3=$ moderately like self, $4=$ very like self). High scores indicate greater interpersonal sensitivity. The psychometric properties of the scale were tested in non-clinical individuals, general practice attenders, and psychiatric patients.

\section{Reasoning}

Cognitive flexibility (Martin $\mathcal{E}$ Rubin, 1995). This is a 12-item self-report scale assessing awareness that in any given situation there are options and alternatives, and the willingness and confidence to be flexible. Items are scores on a six-point scale (strongly agree to strongly disagree). Higher scores indicate greater levels of flexibility. Reliability and validity were established in a non-clinical sample.

Probabilistic reasoning (Garety et al. 2005). Jumping to conclusions was assessed with a probabilistic reasoning task known as the 'beads task'. Participants are shown a jar with 60 black beads and 40 yellow beads ('the mainly black jar') and a jar with 40 black beads and 60 yellow beads ('the mainly yellow jar'). The jars are then hidden from view and the participant told that one of the jars has been selected by the experimenter. The participant is asked to request as many coloured beads as they would like before 
deciding from which of the two hidden jars the beads are drawn. The key variable used here is the number of beads requested before making a decision.

\section{Anomalous experience}

Cardiff Anomalous Perceptions Scale (CAPS; Bell et al. 2006). This 32-item questionnaire, developed in both non-clinical and psychosis groups, assesses perceptual anomalies such as changes in levels of sensory intensity, distortion of the external world, sensory flooding, and hallucinations. A higher score represents the reporting of a greater number of perceptual anomalies. The scale also has three factor scores. The first factor, temporal lobe experience, contains items such as 'Do you ever think that everyday things look abnormal to you?' and 'Do you ever see shapes, lights, or colours even though there is nothing really there?' The second factor, chemosensation, contains items such as 'Do you ever notice that food or drink seems to have an unusual taste' and 'Do you ever smell everyday odours and think that they are unusually strong?' The third factor, clinical psychosis, contains items such as 'Do you ever hear your own thoughts spoken aloud in your head, so that someone near might be able to hear them?' and 'Do you ever hear voices commenting on what you are thinking or doing?'

Maudsley Addiction Profile (MAP; Marsden et al. 1998). The MAP was developed with a large sample from a substance abuse clinic. Respondents are asked directly about the use over the past month of illicit drugs, including cannabis, cocaine powder, crack cocaine, heroin, amphetamines and methadone.

\section{Social}

Life Stressor Checklist (Wolfe \& Kimerling, 1997). The checklist asks respondents about the occurrence of a range of severe life events (e.g. serious accident, physical attack, sexual abuse). If the respondent reports the occurrence of an event, subsequent questions ask when the event happened, whether the person thought at the time serious harm or death could result, and whether feelings of intense helplessness, fear or horror occurred. Only events that reached the severity criterion related to posttraumatic stress disorder diagnosis were scored. The total number of traumatic events, the total number of victimization events, the number of childhood traumatic events, and the number of traumatic events in the past year were recorded. The psychometric properties of the measure are reported by $\mathrm{McHugo}$ et al. (2005).

Social Support Questionnaire (SSQ; Sarason et al. 1987). The short-form of the well-established SSQ (Sarason et al. 1983) was used. Each of the seven items has two parts. The first part assesses the number of people the respondent believes they can turn to in times of need (e.g. 'Whom can you really count on to be dependable when you need help?'). The second part measures the degree of satisfaction with that support. Two scores are derived: the number or perceived availability score and the satisfaction score. Higher scores indicate greater perceptions of social support.

Social and Emotional Loneliness Scale for Adults (DiTommaso $\mathcal{E}$ Spinner, 1993). This 37-item selfreport questionnaire, developed in a non-clinical sample, has three subscales: romantic, family, and social loneliness. Each item is rated on a seven-point scale (ranging from strongly disagree to strongly agree). Higher scores indicate greater levels of loneliness.

\section{Measures of the virtual reality experience}

After being in the virtual environment, participants completed self-report measures of persecutory thinking and social anxiety and visual analogue rating scales.

State Social Paranoia Scale (SSPS; Freeman et al. 2007). The SSPS has 10 persecutory items each rated on a five-point scale (e.g. 'Someone stared at me in order to upset $\mathrm{me}^{\prime}$, 'Someone was trying to isolate $\mathrm{me}^{\prime}$, 'Someone was trying to make me distressed'). The items conform to a recent definition of persecutory ideation (Freeman \& Garety, 2000). Scores can range from 10 (no paranoia) to 50. The SSPS has excellent internal reliability, adequate test-retest reliability, convergent validity with both independent interviewer ratings and self-report measures, and divergent validity with regard to measures of positive and neutral thinking. In the current study the internal reliability of the questionnaire was high (Cronbach's $\alpha=0.90$ ). A person was classified as having (at least some) paranoid thinking if they endorsed one of the paranoid items (i.e. scored 11 or above).

Social Avoidance and Distress Scale (SAD; Watson $\mathcal{E}$ Friend, 1969). The SAD was designed to assess social anxiety. A True-False format is used for each item. 
Higher scores indicate higher levels of social anxiety. Participants were asked to fill in the questionnaire with reference to their experience in the virtual room. SAD items were reworded where necessary. For example, the item 'I usually feel relaxed when I am with a group of people' was changed to 'I felt relaxed with the group of people'. Based upon the original paper it was decided $a$ priori that a score $>7$ (which was the median) would indicate the presence of social anxiety.

Visual analogue rating scales (VAS). To check the validity of the post-virtual reality assessments, participants also marked on two separate $10-\mathrm{cm}$ lines the degree to which the people on the tube were experienced as hostile and how socially anxious the participant felt. Higher ratings indicated greater endorsement of the characteristic.

\section{Analysis}

Analyses were carried out using Stata version 9 (StataCorp, 2005). To look at differences in predictors of the occurrence of paranoia and social anxiety, two binary outcomes were created for each participant from the SSPS and the SAD: the presence of paranoia ( $>10$ SSPS score) and the presence of social anxiety ( $>7$ SAD score). Binary outcomes were created due to the skewed distributions of the dependent variables. The data file was structured so that there were two records for each participant. The first contained all of the covariate information together with the binary indicator of paranoia. The second contained the same covariate information together with the binary indicator of social anxiety. The binary outcomes indicating paranoia and anxiety shared the same variable name but a further variable (Type) was created to indicate whether the record corresponded to paranoia or to anxiety (Type $=1$ for paranoia and Type $=2$ for anxiety). To analyse such a binary bivariate outcome, the marginal modelling technique bivariate logistic regression was used (Fitzmaurice et al. 1995; Dunn, 2000), linking the two records for each participant by a cluster variable specified to be the participant's ID number. The main advantage of the bivariate logistic design is that a single regression model is fitted, instead of two separate regression models being used, which would create separate coefficients that are not easily comparable and would not allow for the regressions being measured on the same individual.

In the analysis each predictor was modelled separately as an interaction with the indicator variable Type. This is the key test for the study and indicates
Table 1. Demographic data

\begin{tabular}{|c|c|}
\hline Variable & Number \\
\hline \multicolumn{2}{|l|}{ Sex } \\
\hline Male & 100 \\
\hline Female & 100 \\
\hline \multicolumn{2}{|l|}{ Ethnicity } \\
\hline White & 135 \\
\hline Black Caribbean & 18 \\
\hline Black African & 9 \\
\hline Black Other & 5 \\
\hline Indian & 6 \\
\hline Pakistani & 1 \\
\hline Other & 26 \\
\hline \multicolumn{2}{|l|}{ Highest education level achieved } \\
\hline None & 11 \\
\hline General Certificate of Secondary Education & 39 \\
\hline Advanced subsidiary/Advanced level & 30 \\
\hline Diploma/Foundation & 27 \\
\hline Degree & 55 \\
\hline Postgraduate diploma & 34 \\
\hline Doctoral degree & 4 \\
\hline \multicolumn{2}{|l|}{ Socio-economic classification (National figure) } \\
\hline Higher professional occupations & $16(11.1 \%)$ \\
\hline $\begin{array}{l}\text { Lower managerial and professional } \\
\text { occupations }\end{array}$ & $57(22.4 \%)$ \\
\hline Intermediate occupations & $17(10.0 \%)$ \\
\hline Small employers and own account workers & $12(7.6 \%)$ \\
\hline $\begin{array}{l}\text { Lower supervisory and technical } \\
\text { occupations }\end{array}$ & $8(9.1 \%)$ \\
\hline Semi-routine occupations & $17(12.8 \%)$ \\
\hline Routine occupations & $13(9.3 \%)$ \\
\hline Never worked and long-term unemployed & $33(3.8 \%)$ \\
\hline Not classifiable (students) & $27(13.7 \%)$ \\
\hline \multicolumn{2}{|l|}{ Frequency of use of underground train } \\
\hline None & 44 \\
\hline Less than monthly & 30 \\
\hline Monthly & 38 \\
\hline Weekly & 70 \\
\hline Daily & 18 \\
\hline \multicolumn{2}{|l|}{$\begin{array}{l}\text { Frequency of playing } \\
\text { computer games }\end{array}$} \\
\hline Never & 101 \\
\hline Once or twice & 51 \\
\hline Once a week & 19 \\
\hline Most days & 24 \\
\hline Every day & 5 \\
\hline
\end{tabular}

whether the effect of the given putative predictor is different for social anxiety and paranoia. Within the Type variable paranoia was always set as the reference category, hence the interaction value represents the interaction with social anxiety, or the change in effect between social anxiety and paranoia. In each case the direct (unadjusted) effects are first reported. 
Table 2. Assessment scores

\begin{tabular}{lrrrc}
\hline & Mean & S.D. & Minimum & Maximum \\
\hline Anxiety & 4.5 & 5.1 & 0 & 31 \\
Depression & 7.2 & 8.6 & 0 & 39 \\
Penn State Worry & 45.7 & 14.8 & 16 & 80 \\
Worry domain & 29.4 & 18.8 & 25 & 108 \\
Negative self & 2.3 & 2.9 & 0 & 15 \\
Positive self & 14.4 & 4.7 & 3 & 24 \\
Negative other & 4.3 & 4.7 & 0 & 22 \\
Positive other & 11.2 & 4.6 & 0 & 24 \\
Cognitive flexibility & 56.6 & 7.3 & 32 & 72 \\
Perceptual anomalies & 7.4 & 6.2 & 0 & 26 \\
Number of lifetime traumas & 2.2 & 2.4 & 0 & 13 \\
Number of social supports & 3.8 & 2.0 & 0.2 & 9.0 \\
Satisfaction with social support & 5.2 & 0.9 & 1.3 & 6.0 \\
\hline
\end{tabular}

S.D., Standard deviation.

The unadjusted analysis will show the direct relationship between the predictor and the outcome; however, these direct relationships may be caused by an extraneous variable or confounder. Confounders are variables that are associated with the predictors and also influence the outcome (response). Failure to allow for this in the statistical analysis leads to distortions (bias) in the estimate of the effect of the predictors. Therefore, also reported are the adjusted effects for a set of constant covariates, which comprised age, sex, ethnicity, IQ, education, use of computer games, use of the London underground, socio-economic status, PSWQ, catastrophic worry interview, cognitive flexibility, anomalous experiences, illicit drug use, and 'Jumping to Conclusions'. The covariates were deemed a priori to be clinically important predictors of paranoia and/or social anxiety. To achieve a set of fully adjusted effects the model included the main effects of each confounder and their interaction with Type effect. All hypothesis testing was two-tailed. Odds ratios (ORs) and 95\% confidence intervals (CIs) are reported.

\section{Results}

\section{Demographic and clinical data}

The average age of the participants was 37.5 years (S.D. $=13.3$, minimum $=18$, maximum $=77$ ). The mean IQ score was 104.6 (S.D. $=12.0$, minimum $=69$, maximum $=133$ ). Further basic information on the participants is presented in Table 1. There is a spread of participants across socio-economic categories, and the proportion in each category is broadly representative of the UK population. Table 2 shows that there is a good range in the symptom scores of the participant group.

\section{Social anxiety and paranoia}

The visual analogue ratings were used to validate the classifications from the SSPS and SAD. Individuals with social anxiety (mean $=4.4$, S.D. $=2.8$ ) scored higher on the VAS of social anxiety than individuals without social anxiety (mean $=1.2$, S.D. $=1.6, t=-7.6$, $\mathrm{df}=57.2, \quad p<0.001)$. Individuals with paranoia $($ mean $=2.3$, S.D. $=1.9)$ scored higher on the VAS of hostility by the computer characters than the individuals without paranoia (mean $=0.8$, s.D. $=1.4$, $t=5.9, \mathrm{df}=170.6, p<0.001)$. Ninety-three people had no paranoia or social anxiety, 59 people had paranoia and social anxiety, 36 people had paranoia without social anxiety and 12 people had social anxiety without paranoia.

\section{Differential predictors}

The differential predictor analysis is reported in Table 3. For the interpretation of the results it should be noted that for continuous scales the ORs refer to 1-point changes; if the OR for a unit change in the independent variable is, for example, 1.10 then the OR for a 10-point increase is 1.10 raised to the power of 10 (i.e. 2.59). For unadjusted effects, only one variable had a significant interaction, Positive Self ( $p$ value $=0.006)$. As positive self increases by 1 unit the odds decrease by 0.97 for paranoia and 0.87 $(0.97 \times 0.90)$ for social anxiety.

The unadjusted effects do not take into account any confounding caused by other factors and hence true effects may be masked or misleading. Table 3 also displays the effects produced for each predictor when adjusted for the set of potential confounders. Two clinical variables, anomalies of experience and family loneliness, significantly differed in their 
Table 3. The differential prediction of anxiety and paranoia

\begin{tabular}{|c|c|c|c|c|c|c|}
\hline \multirow[b]{2}{*}{ Variable } & \multicolumn{3}{|c|}{ Unadjusted (direct) } & \multicolumn{3}{|c|}{ Adjusted } \\
\hline & OR & $p$ value & $95 \% \mathrm{CI}$ & OR & $p$ value & $95 \% \mathrm{CI}$ \\
\hline Age & 0.99 & 0.631 & $0.97-1.02$ & 1.01 & 0.722 & $0.97-1.04$ \\
\hline Type $\times$ Age & 0.98 & 0.130 & $0.95-1.01$ & 0.98 & 0.468 & $0.94-1.03$ \\
\hline IQ & 1.00 & 0.706 & $0.98-1.03$ & 1.04 & 0.039 & $1.00-1.08$ \\
\hline Type × IQ & 1.00 & 0.768 & $0.97-1.04$ & 0.99 & 0.651 & $0.95-1.03$ \\
\hline Anxiety & 1.11 & 0.000 & $1.05-1.18$ & 1.05 & 0.345 & $0.95-1.15$ \\
\hline Type $\times$ Anxiety & 0.97 & 0.361 & $0.89-1.04$ & 1.00 & 0.938 & $0.89-1.11$ \\
\hline Penn State Worry & 1.03 & 0.006 & $1.01-1.05$ & 1.03 & 0.026 & $1.00-1.06$ \\
\hline Type $\times$ WorryPenn & 1.02 & 0.132 & $0.99-1.05$ & 1.01 & 0.507 & $0.98-1.05$ \\
\hline Worry Domain & 1.03 & 0.000 & $1.02-1.05$ & 1.00 & 0.760 & $0.98-1.03$ \\
\hline Type $\times$ WorryDom & 1.01 & 0.251 & $0.99-1.03$ & 1.04 & 0.067 & $1.00-1.07$ \\
\hline Worry Catastrophic & 1.05 & 0.035 & $1.00-1.09$ & 1.05 & 0.063 & $1.00-1.10$ \\
\hline Type $\times$ WorryCat & 1.00 & 0.855 & $0.95-1.04$ & 1.00 & 0.942 & $0.95-1.06$ \\
\hline Interpersonal Sensitivity & 1.04 & 0.001 & $1.01-1.06$ & 1.02 & 0.170 & $0.99-1.06$ \\
\hline Type $\times$ Sensitivity & 1.01 & 0.614 & $0.98-1.04$ & 1.00 & 0.899 & $0.97-1.04$ \\
\hline Negative Self & 1.20 & 0.002 & $1.07-1.34$ & 1.03 & 0.763 & $0.86-1.22$ \\
\hline Type $\times$ NegSelf & 1.02 & 0.743 & $0.91-1.15$ & 1.08 & 0.382 & $0.91-1.30$ \\
\hline Positive Self & 0.97 & 0.269 & $0.91-1.03$ & 1.03 & 0.597 & $0.92-1.15$ \\
\hline Type $\times$ PosSelf & 0.90 & $0.006^{* *}$ & $0.83-0.97$ & 0.89 & 0.076 & $0.78-1.01$ \\
\hline Negative Other & 1.08 & 0.019 & $1.01-1.15$ & 1.03 & 0.515 & $0.93-1.14$ \\
\hline Type $\times$ NegOther & 0.96 & 0.255 & $0.89-1.03$ & 0.93 & 0.335 & $0.81-1.07$ \\
\hline Positive Other & 0.97 & 0.271 & $0.91-1.03$ & 1.03 & 0.460 & $0.95-1.13$ \\
\hline Type $\times$ PosOther & 0.95 & 0.188 & $0.88-1.03$ & 0.92 & 0.109 & $0.84-1.02$ \\
\hline Depression & 1.06 & 0.006 & $1.02-1.10$ & 1.03 & 0.362 & $0.97-1.10$ \\
\hline Type $\times$ Dep & 1.00 & 0.993 & $0.96-1.04$ & 1.00 & 0.932 & $0.93-1.07$ \\
\hline Cognitive Flexibility & 0.96 & 0.058 & $0.93-1.00$ & 0.98 & 0.438 & $0.92-1.04$ \\
\hline Type $\times$ Cogflex & 0.97 & 0.142 & $0.92-1.01$ & 0.97 & 0.341 & $0.91-1.03$ \\
\hline Jumping to Conclusions & 1.00 & 0.941 & $0.94-1.06$ & 1.04 & 0.414 & $0.95-1.13$ \\
\hline Type $\times$ JTC & 1.02 & 0.701 & $0.94-1.10$ & 0.95 & 0.458 & $0.84-1.08$ \\
\hline Anomalous Perceptions Scale & 1.07 & 0.003 & $1.02-1.12$ & 1.09 & 0.006 & $1.02-1.16$ \\
\hline Type $\times$ Anomalous & 0.95 & 0.084 & $0.90-1.01$ & 0.91 & $0.007^{* *}$ & $0.85-0.97$ \\
\hline Romantic Loneliness & 1.01 & 0.170 & $1.00-1.02$ & 1.00 & 0.928 & $0.98-1.02$ \\
\hline Type $\times$ Romloneliness & 1.00 & 0.914 & $0.98-1.02$ & 1.00 & 0.892 & $0.98-1.03$ \\
\hline Family Loneliness & 1.03 & 0.009 & $1.01-1.05$ & 1.00 & 0.775 & $0.97-1.04$ \\
\hline Type $\times$ Famloneliness & 1.01 & 0.218 & $0.99-1.04$ & 1.04 & $0.024^{*}$ & $1.00-1.07$ \\
\hline Social Loneliness & 1.02 & 0.035 & $1.00-1.04$ & 1.00 & 0.704 & $0.98-1.03$ \\
\hline Type $\times$ SocLone & 1.01 & 0.556 & $0.99-1.03$ & 1.01 & 0.334 & $0.99-1.04$ \\
\hline Support Satisfaction & 0.61 & 0.013 & $0.42-0.90$ & 0.79 & 0.396 & $0.45-1.37$ \\
\hline Type $\times$ SupSat & 0.95 & 0.788 & $0.63-1.42$ & 0.71 & 0.180 & $0.43-1.17$ \\
\hline No. of Social Supports & 0.87 & 0.044 & $0.76-1.00$ & 0.92 & 0.431 & $0.75-1.13$ \\
\hline Type $\times$ NoSocial & 1.14 & 0.187 & $0.94-1.37$ & 1.13 & 0.375 & $0.86-1.48$ \\
\hline Number of Lifetime Trauma & 1.12 & 0.091 & $0.98-1.27$ & 1.01 & 0.904 & $0.83-1.23$ \\
\hline Type $\times$ Lifetrauma & 0.97 & 0.702 & $0.83-1.14$ & 1.06 & 0.568 & $0.86-1.31$ \\
\hline Number Lifetime Victim & 1.07 & 0.475 & $0.88-1.30$ & 0.96 & 0.778 & $0.74-1.25$ \\
\hline Type $\times$ LifeVictimization & 1.07 & 0.495 & $0.87-1.32$ & 1.32 & 0.076 & $0.97-1.81$ \\
\hline Childhood abuse present & 1.49 & 0.223 & $0.79-2.83$ & 0.92 & 0.848 & $0.39-2.16$ \\
\hline Type $\times$ Childabuse & 0.95 & 0.887 & $0.44-2.03$ & 1.99 & 0.201 & $0.69-5.72$ \\
\hline Number Recent Trauma & 1.37 & 0.276 & $0.78-2.43$ & 1.08 & 0.828 & $0.53-2.22$ \\
\hline Type $\times$ Recenttrauma & 0.70 & 0.339 & $0.34-1.45$ & 0.68 & 0.428 & $0.26-1.76$ \\
\hline Sense of presence & 0.98 & 0.577 & $0.93-1.04$ & 0.99 & 0.863 & $0.92-1.08$ \\
\hline Type $\times$ Senseofpresence & 1.05 & 0.107 & $0.99-1.12$ & 1.03 & 0.548 & $0.94-1.12$ \\
\hline Illicit drug use & 1.75 & 0.066 & $0.96-3.19$ & 1.62 & 0.264 & $0.70-3.78$ \\
\hline Type $\times$ Illicitdruguse & 0.82 & 0.618 & $0.38-1.76$ & 1.29 & 0.602 & $0.50-3.31$ \\
\hline
\end{tabular}

OR, Odds ratio; $\mathrm{CI}$, confidence interval.

Interaction effects: ${ }^{*} p<0.05,{ }^{* *} p<0.01$. 
Table 4. Differential prediction using the subscales of the anomalous perceptual experiences scale

\begin{tabular}{|c|c|c|c|c|c|c|}
\hline \multirow[b]{2}{*}{ Variable } & \multicolumn{3}{|c|}{ Unadjusted (direct) } & \multicolumn{3}{|c|}{ Adjusted } \\
\hline & OR & $p$ value & $95 \%$ CI & OR & $p$ value & $95 \% \mathrm{CI}$ \\
\hline Temporal lobe & 1.17 & 0.011 & $1.04-1.32$ & 0.90 & 0.575 & $0.63-1.29$ \\
\hline Type $\times$ Templobe & 0.92 & 0.265 & $0.79-1.07$ & 1.18 & 0.474 & $0.75-1.84$ \\
\hline Chemosensation & 1.14 & 0.027 & $1.02-1.29$ & 0.91 & 0.605 & $0.63-1.31$ \\
\hline Type $\times$ Chemosensation & 0.85 & $0.041^{*}$ & $0.73-0.99$ & 0.79 & 0.322 & $0.50-1.25$ \\
\hline Clinical psychosis & 1.54 & 0.008 & $1.12-2.13$ & 1.07 & 0.825 & $0.60-1.91$ \\
\hline Type $\times$ Clinpsychosis & 0.75 & 0.130 & $0.52-1.09$ & 1.19 & 0.648 & $0.56-2.54$ \\
\hline Nonpsychosis anomalies & 1.12 & 0.011 & $1.03-1.23$ & 0.97 & 0.852 & $0.73-1.30$ \\
\hline Type $\times$ Nonpsychosis & 0.91 & 0.105 & $0.80-1.02$ & 0.81 & 0.197 & $0.59-1.11$ \\
\hline
\end{tabular}

OR, Odds ratio; $\mathrm{CI}$, confidence interval.

Interaction effects: ${ }^{*} p<0.05$.

relationships with paranoia and social anxiety. Anomalies of experience is highly statistically significant $(p=0.007)$, indicating that the effect of anomalous experience is different for paranoia and social anxiety. The effect is represented as an OR for paranoia of 1.09 but for social anxiety $0.99(1.09 \times 0.91)$. This means that, within paranoia, the odds increase by 1.09 as the anomalies of experience scale increases by 1 unit; however, within social anxiety the odds decrease by 0.99 as the scale increases by 1 unit. An increase on the family loneliness scale does not affect the occurrence of paranoia but increases the odds of social anxiety by 1.04 .

The CAPS assesses a wide range of phenomena, from subtle perception distortions to auditory hallucinations. It was of interest to determine whether it was only the presence of clinical psychosis-like symptoms that separated the two experiences. In a post hoc analysis, the analysis was repeated using the three factors of the scale (temporal lobe epilepsy, chemosensation, clinical psychosis) and also a variable that comprised items that could not be thought of as psychosis-like symptoms (items 1, 15, 16, 17, $18,20,21,22,23,25,26,30)$. Table 4 shows that the differential relationship is not simply explained by the presence of clinical psychosis symptoms. The unadjusted affects do show a relationship for the subscale Chemosensation ( $p$ value $=0.041$ ). In this case the OR is calculated to be 1.14 within paranoia but $0.97(1.14 \times 0.85)$ within the social anxiety group.

\section{Discussion}

This is the first study to examine rigorously the differential prediction of social anxiety and persecutory ideation. Using a sophisticated statistical analysis, the relationships of psychological and social variables to well-established measures of paranoia and social anxiety were tested in a non-clinical population. The results are intriguing. Foremost, it was the assessment of perceptual anomalies that differentially predicted paranoid and social anxiety reactions. Individuals with paranoid reactions were prone to internal anomalous experience, whereas individuals with social anxiety reactions were less prone to internal anomalous experience. It was not simply psychosis-like anomalies but a wide range of perceptual experiences, from the mild to the severe, that predicted psychological reactions. For instance, items in the chemosensation subscale, which mainly relate to olfactory and gustatory experiences, include: 'Do you ever smell everyday odours and think that they are unusually strong?', 'Do you ever think that food or drink tastes much stronger than it normally would?', 'Do you ever find that your skin is more sensitive to touch, heat or cold than usual?' The importance of perceptual anomalies to paranoia is consistent with the cognitive model of paranoia. Having odd internal feelings in social situations may lead to delusional ideas through a sense of 'things not seeming right'. However, an important caveat is that the nature of the association of paranoia and perceptual anomalies was not established in the study. Anomalies at the time of testing were not assessed. A causal role can only be established in a study that manipulates anomalies of experience, in a randomized controlled design similar to the report by Zimbardo et al. (1981). Such causal designs are now indicated in research on the psychology of psychosis.

The emphasis in the report has been on identifying differences between anxious and suspicious thinking. However, the absence of differences for the majority of the variables is a striking finding. Participants' levels of anxiety, depression, worry and interpersonal sensitivity had similar relationships to both social anxiety and paranoia. This very much confirms recent 
ideas about the contribution of anxiety to paranoid experience, but challenges the more traditional view of clear-cut distinctions between psychotic and emotional problems. Paranoia can be conceptualized as a type of anxious fear. The clinical implication is that approaches used to treat anxiety disorders, suitably modified, will also be of benefit to people with paranoia (Freeman et al. 2006). This is an emerging research strategy that focuses on experiences such as paranoid thoughts, not on diagnoses such as schizophrenia, and treats problems as on a continuum of severity in the population.

There were a number of limitations to the study that should be kept in mind. Multiple hypothesis testing was carried out, raising the likelihood of the occurrence of Type I errors, although the results were broadly consistent with the current theoretical understanding of paranoia. Furthermore, the dependent variables had considerable skew, leading to their dichotomization and a reduction in statistical power. Inevitably the identification of paranoid thinking and social anxiety depends on self-report. The study therefore relied on people being able to report their thoughts from the time spent in virtual reality. The participants were volunteers, responding to a leaflet distributed to local postcodes, who did not know beforehand that they were taking part in a study of paranoia. They were broadly representative of the UK in terms of employment status. However, this recruitment method is unlikely to have resulted in a truly representative sample of the population being tested. It is also clear that this is a study of low-level persecutory and anxious thinking. Similar studies looking at differential prediction in more severe instances would be of great interest.

\section{Acknowledgements}

This research was supported by a Wellcome Trust Fellowship awarded to D.F.

\section{Declaration of Interest}

None.

\section{References}

Bell V, Halligan PW, Ellis HD (2006). The Cardiff Anomalous Perceptions Scale (CAPS). Schizophrenia Bulletin 32, 366-377.

Birchwood M (2003). Pathways to emotional dysfunction in first episode psychosis. British Journal of Psychiatry 182, 373-375.
Boyce P, Parker G (1989). Development of a scale to measure interpersonal sensitivity. Australian and New Zealand Journal of Psychiatry 23, 341-351.

Butler PD, Javitt DC (2005). Early-stage visual processing deficits in schizophrenia. Current Opinion in Psychiatry 18, 151-157.

Chapman LJ, Chapman JP (1980). Scales for rating psychotic and psychotic-like experiences as continua. Schizophrenia Bulletin 6, 476-489.

Chapman LJ, Chapman JP, Kwapil TR, Eckbald M, Zinser MC (1994). Putatively psychosis-prone subjects 10 years later. Journal of Abnormal Psychology 103, 171-183.

Claridge G (1994). Single indicators of risk for schizophrenia. Probably fact or likely myth? Schizophrenia Bulletin 20, 151-168.

Claridge G (1997). Schizotypy: Implications for Illness and Health. Oxford University Press: Oxford.

Crawford JR, Henry JD (2003). The Depression Anxiety Stress Scales (DASS) : normative data and latent structure in a large non-clinical sample. British Journal of Clinical Psychology 42, 111-131.

DiTommaso E, Spinner B (1993). The development and initial validation of the social and emotional loneliness scale for adults. Personality and Individual Differences 14, 127-134.

D'Souza DC, Perry E, MacDougall L, Ammerman Y, Cooper T, Wu Y, Braley G, Gueorguieva R, Krystal JH (2004). The psychotomimetic effects of intravenous delta 9-tetrahydrocannabinol in healthy individuals: implications for psychosis. Neuropsychopharmacology 29, 1558-1572.

Dunn G (2000). Statistics in Psychiatry. Arnold: London.

Eaton WW, Romanoski A, Anthony JC, Nestadt G (1991). Screening for psychosis in the general population with a self-report interview. Journal of Nervous and Mental Disease 179, 689-693.

Fitzmaurice GM, Laird NM, Zahner GEP, Daskalakis C (1995). Bivariate logistic regression analysis of childhood psychopathology ratings using multiple informants. American Journal of Epidemiology 142, 1194-1203.

Fowler D, Freeman D, Smith B, Kuipers E, Bebbington P, Bashforth H, Coker S, Gracie A, Dunn G, Garety P (2006). The Brief Core Schema Scales (BCSS): psychometric properties and associations with paranoia and grandiosity in non-clinical and psychosis samples. Psychological Medicine 36, 749-759.

Frecon E, Smith G, Steed A, Stenius M, Stahl O (2001). An overview of the COVEN platform. Presence: Teleoperators and Virtual Environments 10, 109-127.

Freeman D (2007). Suspicious minds: the psychology of persecutory delusions. Clinical Psychology Review 27, 425-457.

Freeman D (2008a). Studying and treating schizophrenia using virtual reality: a new paradigm. Schizophrenia Bulletin. Published online: 28 March 2008. doi: 10.1093/ schbul/sbn020.

Freeman D (2008b). The assessment of persecutory ideation. In Persecutory Delusions (ed. D. Freeman, R. Bentall and P. Garety). Oxford University Press: Oxford. 
Freeman D, Freeman J (2008). Paranoia: The 21st Century Fear. Oxford University Press: Oxford.

Freeman D, Freeman J, Garety P (2006). Overcoming Paranoid and Suspicious Thoughts. Robinson Constable: London.

Freeman D, Garety PA (1999). Worry, worry processes and dimensions of delusions: an exploratory investigation of a role for anxiety processes in the maintenance of delusional distress. Behavioural \& Cognitive Psychotherapy 27, 47-62.

Freeman D, Garety PA (2000). Comments on the content of persecutory delusions: does the definition need clarification? British Journal of Clinical Psychology 39, 407-414.

Freeman D, Garety PA, Bebbington P, Slater M, Kuipers E, Fowler D, Green C, Jordan J, Ray K, Dunn G (2005a). The psychology of persecutory ideation. II. A virtual reality experimental study. Journal of Nervous and Mental Disease 193, 309-315.

Freeman D, Garety PA, Bebbington PE, Smith B, Rollinson R, Fowler D, Kuipers E, Ray K, Dunn G (2005b). Psychological investigation of the structure of paranoia in a non-clinical population. British Journal of Psychiatry 186, 427-435.

Freeman D, Garety PA, Fowler D, Kuipers E, Bebbington P, Dunn G (2004). Why do people with delusions fail to choose more realistic explanations for their experiences? An empirical investigation. Journal of Consulting and Clinical Psychology 72, 671-680.

Freeman D, Garety PA, Kuipers E, Fowler D, Bebbington PE (2002). A cognitive model of persecutory delusions. British Journal of Clinical Psychology 41, 331-347.

Freeman D, Pugh K, Antley A, Slater M, Bebbington P, Gittins M, Dunn G, Kuipers E, Fowler D, Garety P (2008). A virtual reality study of paranoid thinking in the general population. British Journal of Psychiatry 192, 258-263.

Freeman D, Pugh K, Green C, Valmaggia L, Dunn G, Garety P (2007). A measure of state persecutory ideation for experimental studies. Journal of Nervous and Mental Disease 195, 781-784.

Freeman D, Slater M, Bebbington PE, Garety PA, Kuipers E, Fowler D, Met A, Read C, Jordan J, Vinayagamoorthy V (2003). Can virtual reality be used to investigate persecutory ideation? Journal of Nervous and Mental Disease 191, 509-514.

Garety PA, Bebbington P, Fowler D, Freeman D, Kuipers E (2007). Implications for neurobiological research of cognitive models of psychosis. Psychological Medicine 37, 1377-1391.

Garety PA, Freeman D (1999). Cognitive approaches to delusions: a critical review of theories and evidence. British Journal of Clinical Psychology 38, 113-154.

Garety PA, Freeman D, Jolley S, Dunn G, Bebbington PE, Fowler D, Kuipers E, Dudley R, (2005). Reasoning, emotions and delusional conviction in psychosis. Journal of Abnormal Psychology 114, 373-384.

Hemsley DR (2005). The schizophrenic experience: taken out of context? Schizophrenia Bulletin 31, 43-53.
Johns LC, Cannon M, Singleton N, Murray RM, Farrell M, Brugha T, Bebbington P, Jenkins R, Meltzer H (2004). The prevalence and correlates of self-reported psychotic symptoms in the British population. British Journal of Psychiatry 185, 298-305.

Kapur S (2003). Psychosis as a state of aberrant salience: a framework linking biology, phenomenology, and pharmacology. American Journal of Psychiatry 160, 13-23.

Lovibond PF, Lovibond SH (1995). The structure of negative emotional states: comparison of the Depression Anxiety Stress Scales (DASS) with the Beck depression and anxiety inventories. Behaviour Research and Therapy 33, 335-343.

Marsden J, Gossop G, Stewart D, Best D, Farrell M, Lehmann P, Edwards C, Strang J (1998). The Maudsley Addition Profile (MAP). Addiction 93, 1857-1867.

Martin JA, Penn DL (2001). Brief report: social cognition and subclinical paranoid ideation. British Journal of Clinical Psychology 40, 261-265.

Martin MM, Rubin RB (1995). A new measure of cognitive flexibility. Psychological Reports 76, 623-626.

McHugo GJ, Caspi Y, Kammerer N, Mazelis R, Jackson EW, Russell L, Clark C, Liebschutz J, Kimerling R (2005). The assessment of trauma history in women with co-occurring substance abuse and mental disorders and a history of interpersonal violence. Journal of Behavioural Health Services and Research 32, 113-127.

Meyer TJ, Miller ML, Metzger RL, Borkovec TD (1990). Development and validation of the Penn State Worry Questionnaire. Behaviour Research and Therapy 28, 487-495.

Myin-Germeys I, Krabbendam L, van Os J (2003). Continuity of psychotic symptoms in the community. Current Opinion in Psychiatry 16, 443-449.

Office for National Statistics (2005). The National Statistics Socio-economic Classification. Palgrave Macmillan: Hampshire.

Poulton R, Caspi A, Moffitt TE, Cannon M, Murray R, Harrington H (2000). Children's self-reported psychotic symptoms and adult schizophreniform disorder. Archives of General Psychiatry 57, 1053-1058.

Sanchez-Vives MV, Slater M (2005). From presence to consciousness through virtual reality. Nature Reviews Neuroscience 6, 332-339.

Sarason IG, Levine HM, Basham RB, Sarason BR (1983). Assessing social support: the Social Support Questionnaire. Journal of Personality and Social Psychology 44, 127-139.

Sarason IG, Sarason BR, Shearin EN, Plerce GR (1987). A brief measure of social support. Journal of Social and Personal Relationships 4, 497-510.

Startup H, Freeman D, Garety PA (2007). Persecutory delusions and catastrophic worry in psychosis: developing the understanding of delusion distress and persistence. Behaviour Research and Therapy 45, 523-537.

Startup HM, Erickson TM (2006). The Penn State Worry Questionnaire. In Worry and Its Psychological Disorders (ed. G. Davey and A. Wells), pp. 101-120. Wiley: Chichester. 
StataCorp (2005). Stata Statistical Software: Release 9.0. Stata Corporation: College Station, TX.

Strauss JS (1969). Hallucinations and delusions as points on continua function. Archives of General Psychiatry 20, 581-586.

Tallis F, Eysenck MW, Mathews A (1992). A questionnaire for the measurement of nonpathological worry. Personality and Individual Differences 13, 161-168.

Valmaggia L, Freeman D, Green C, Garety P, Swapp D, Antley A, Prescott C, Fowler D, Kuipers E, Bebbington P, Slater M, Broome M, McGuire P (2007). Virtual reality and paranoid ideations in people with an 'at risk mental state' for psychosis. British Journal of Psychiatry 191, s63-s68.

Van Dael F, Versmissen D, Janssen I, Myin-Germeys I, van Os J, Krabbendam L (2006). Data gathering: biased in psychosis? Schizophrenia Bulletin 32, 341-351.

Van Os J, Hanssen M, Bijl RV, Ravelli A (2000). Strauss (1969) revisited: a psychosis continuum in the general population. Schizophrenia Research 45, 11-20.

Van Os J, Verdoux H (2003). Diagnosis and classification of schizophrenia: categories versus dimensions, distributions versus disease. In The Epidemiology of Schizophrenia (ed. R. M. Murray, P. B. Jones, E. Susser, J. van Os and M. Cannon), pp. 364-410. Cambridge University Press: Cambridge.

Vasey M, Borkovec TD (1992). A catastrophising assessment of worrisome thoughts. Cognitive Therapy and Research 16, 505-520.

Watson D, Friend R (1969). Measurement of social-evaluative anxiety. Journal of Consulting and Clinical Psychology 33, 448-457.

Wechsler D (1997). Wechsler Adult Intelligence Scale - Third Edition. The Psychological Corporation: San Antonio, TX.

Wechsler D (1999). Wechsler Abbreviated Scale of Intelligence (WASI). The Psychological Corporation: San Antonio, TX.

Wolfe J, Kimerling R (1997). Gender issues in the assessment of posttraumatic stress disorder. In Assessing Psychological Trauma and PTSD (ed. J. Wilson and T. Keane), pp. 192-238. Guilford Press: New York. Zimbardo PG, Andersen SM, Kabat LG (1981). Induced hearing deficit generates experimental paranoia. Science 212, 1529-1531. 